\title{
Image Retrieval with Shape Features Extracted using Gradient Operators and Slope Magnitude Technique with BTC
}

\author{
Dr.H.B.Kekre ${ }^{1}$ \\ Sudeep Thepade ${ }^{2}$ \\ ${ }^{1}$ Sr.Professor, ${ }^{2}$ Associate Professor, \\ Priyadarshini Mukherjee, \\ Miti Kakaiya, \\ B.Tech Student, \\ MPSTME, SVKM's NMIMS University, MPSTME, SVKM's NMIMS University, MPSTME, SVKM's NMIMS University, \\ Mumbai \\ Shobhit Wadhwa, \\ Satyajit Singh, \\ B.Tech Student, \\ Mumbai
}

\begin{abstract}
The paper discusses novel image retrieval methods based on shape features extracted using gradient operators and slope magnitude technique with Block Truncation Coding (BTC). Four variations of proposed 'Mask-Shape-BTC' image retrieval techniques are proposed using gradient masks like Robert, Sobel, Prewitt and Canny. The proposed image retrieval techniques are tested on generic image database with 1000 images spread across 11 categories. In all 55 queries (5 from each category) are fired on the image database. The average precision and recall of all queries are computed and considered for performance analysis. In all the considered gradient operators for shape extraction, 'Mask-ShapeBTC' CBIR techniques outperform the 'Mask-Shape' CBIR techniques. The performance ranking of the masks for proposed image retrieval methods can be listed as Robert (best performance), Prewitt, Sobel and lastly the Canny.
\end{abstract}

\section{Keywords}

CBIR, Shape Feature, Sobel, Prewitt, Robert, Canny, Slope Magnitude, BTC.

\section{INTRODUCTION}

Information retrieval (IR) is the science of searching for documents, for information within documents, and for metadata about documents, as well as that of searching relational databases and the World Wide Web. There is overlap in the usage of the terms data retrieval, document retrieval, information retrieval, and text retrieval, but each also has its own body of literature, theory and technologies. IR is interdisciplinary, based on computer science, mathematics, cognitive psychology, linguistics, statistics, and physics. Automated information retrieval systems are used to reduce what has been called "information overload". Many universities and public libraries use IR systems to provide access to books and journals. Web search engines are the most visible IR applications. Images do have giant share in this information being stored and retrieved.

\subsection{Image Retrieval}

Image search is a specialized data search used to find images. User may give a keyword, sketch or an image to image search engine for retrieving the relatively similar images from the image databases. The similarity used for search criteria could be meta tags, color distribution in images and region/shape attributes. Most traditional methods of image retrieval utilize some method of adding metadata such as captioning, keywords, or descriptions to the images so that retrieval can be performed over the annotation words. [23]. The limitations of text-based approach are that it is subject to human perception and the problem of annotation of images. Annotating every image is a cumbersome and expensive task.

Content-based image retrieval (CBIR) is the application of computer vision to the image retrieval problem, that is, the problem of searching for digital images in large databases. The term 'content' in this context might refer to color, shapes, textures. The color aspect can be achieved by the techniques averaging and histograms $[4,5,7]$. The texture aspect can be achieved by using transforms [12] or vector quantization [9, 11, 15]. Finally the shape aspect can be achieved by using gradient operators or morphological operators. Some of the major areas of application are: Art collections, Medical diagnosis, Crime prevention, The military, Intellectual property, Architectural and engineering design and Geographical information and remote sensing systems.

\subsection{Content Based Image Retrieval (CBIR)}

The images are very rich in the content like color, tecture, shape information present in them. Retrieving images based on color similarity is achieved by computing a color histogram for each image that identifies the proportion of pixels within an image holding specific values (that humans express as colors). Color searches will usually involve comparing color histograms, though this is not the only technique in practice. Texture measures look for visual patterns in images and how they are spatially defined. The identification of specific textures in an image is achieved primarily by modeling texture as a two-dimensional gray level variation. The relative brightness of pairs of pixels is computed such that degree of contrast, regularity, coarseness and directionality may be estimated. Shape does not refer to the shape of an image but to the shape of a particular region that is being sought out. Shapes will often be determined first applying segmentation or edge detection to an image. Other methods use shape filters to identify given shapes of an image. In some case accurate shape detection will require human intervention because methods like segmentation are very difficult to completely automate. Here the paper discuss shape extraction using edge detection masks like Sobel, Roberts, Prewitt and Canny gradient operators.

\section{EDGE DETECTION MASKS}

Edge detection is a very important in image analysis. As the edges give idea about the shapes of objects present in the image so they are useful for segmentation, registration, and identification of objects in a scene. An edge is a jump in intensity. The cross section of an edge has the shape of a ramp [22, 24, 26]. An ideal 
edge is a discontinuity (i.e., a ramp with an infinite slope). The first derivative assumes a local maximum at an edge. The various gradient operators used for edge extraction are Sobel, Prewitt, Roberts and Canny. Sobel Operator is a discrete differentiation operator, computing an approximation of the gradient of the image intensity function. The Sobel operator is based on convolving the image with a small, separable, and integer valued filter in horizontal and vertical direction and is therefore relatively inexpensive in terms of computations. The Sobel mask can be depicted as shown in equation 1 and 2 .

$$
\begin{gathered}
G_{y}=\left[\begin{array}{ccc}
+1 & +2 & +1 \\
0 & 0 & 0 \\
-1 & -1 & -1
\end{array}\right] \\
G_{x}=\left[\begin{array}{lll}
+1 & 0 & -1 \\
+2 & 0 & -2 \\
+1 & 0 & -1
\end{array}\right]
\end{gathered}
$$

Prewitt edge detector is an appropriate way to estimate the magnitude and orientation of an edge. The Prewitt mask can be defined as given in equations 3 and 4 .

$$
\begin{aligned}
G_{x} & =\left[\begin{array}{ccc}
-1 & 0 & +1 \\
-1 & 0 & +1 \\
-1 & 0 & +1
\end{array}\right] \\
G_{y} & =\left[\begin{array}{ccc}
-1 & -1 & -1 \\
0 & 0 & 0 \\
+1 & +1 & +1
\end{array}\right]
\end{aligned}
$$

Equations 5 and 6 show the Roberts gradient operators. It is used to detect edges applying a horizontal and vertical filter in sequence. Both filters are applied to the image and then slope magnitude method could be applied to extract the connected edges in the image.

$$
\begin{aligned}
G_{x} & =\left[\begin{array}{cc}
+1 & 0 \\
0 & -1
\end{array}\right] \\
G_{y} & =\left[\begin{array}{cc}
0 & +1 \\
-1 & 0
\end{array}\right]
\end{aligned}
$$

Canny edge detector uses a filter based on the first derivative of a Gaussian, because it is susceptible to noise present on raw unprocessed image data, so to begin with, the raw image is convolved with a Gaussian filter. Canny operator is nothing but gradient of Gaussian filtered image.

\section{SLOPE MAGNITUDE METHOD}

The problem with edge extraction using gradient operators is detection of edges in only either horizontal or vertical directions. Shape feature extraction in image retrieval requires the extracted edges to be connected in order to reflect the boundaries of objects present in the image. Slope magnitude method is used along with the gradient operators (Sobel, Prewitt, Robert and Canny) to extract the shape features in form of connected boundaries. The process of applying the slope magnitude method is given as follows. First one needs to convolve the original image with the
Gx mask to get the $\mathrm{x}$ gradient and Gy mask to get the $\mathrm{y}$ gradient of the image. Then the individual squares of both are taken. Finally the two squared terms are added and square root of this sum is taken as given in equation 7 .

$$
G=\sqrt{G_{x}^{2}+G_{y}^{2}}
$$

For the sample image shown in figure 1 the shape features extracted using the gradient operators Sobel, Prewitt, Roberts and Canny are respectively shown in figure 2, 3, 4 and 5.

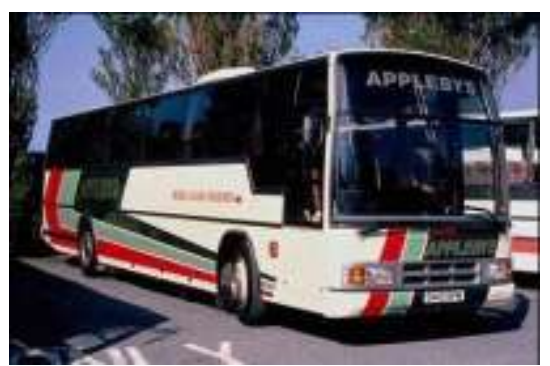

Figure 1: Input Image for Slope Magnitude Technique

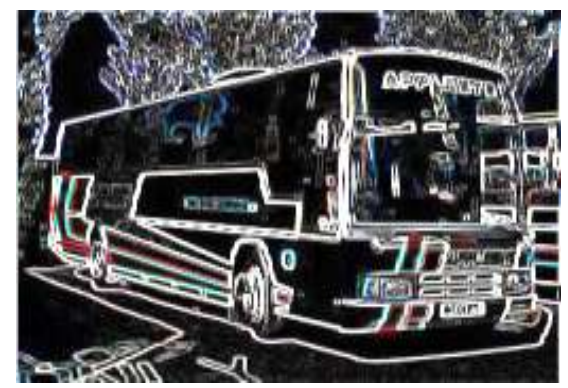

Figure 2: Shape extracted using Slope Magnitude Technique with Canny Operator

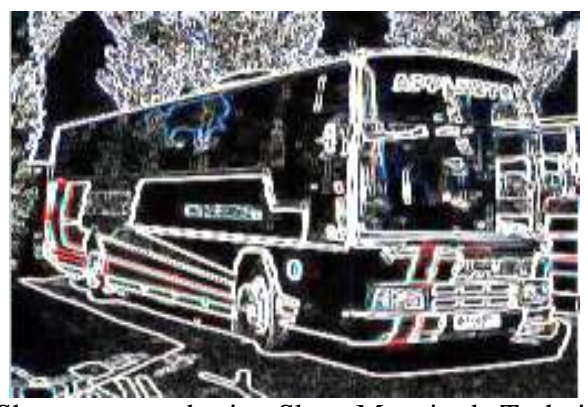

Figure 3: Shape extracted using Slope Magnitude Technique with Sobel Operator

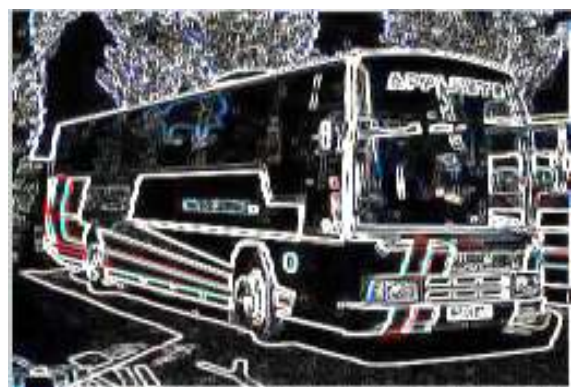

Figure 4: Shape extracted using Slope Magnitude Technique with Prewitt Operator 


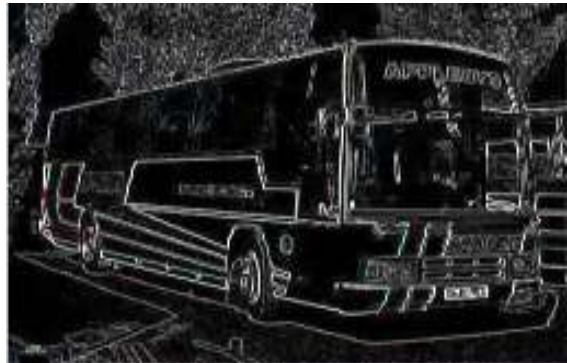

Figure 5: Shape extracted using Slope Magnitude Technique with Robert Operator

\section{BLOCK TRUNCATION CODING (BTC)}

Block truncation coding (BTC) is a simple image coding technique developed in the early years of digital imaging. This method first divides the image to be coded into small nonoverlapping image blocks typically of size $4 \times 4$ pixels to achieve reasonable quality. The small blocks are coded one at a time. For each block, the original pixels within the block are coded using a binary bit-map the same Upper Mean Color (UM) size as the original blocks and two mean pixel values. The method first computes the mean pixel value of the whole block and then each pixel in that block is compared to the block mean. If a pixel is greater than or equal to the block mean, the corresponding pixel position of the bitmap will have a value of 1 otherwise it will have a value of 0 . Two mean pixel values one for the pixels greater than or equal to the block mean and the other for the pixels smaller than the block mean are also calculated. At decoding stage, the small blocks are decoded one at a time. For each block, the pixel positions where the corresponding bitmap has a value of 1 is replaced by one mean pixel value and those pixel positions where the corresponding bitmap has a value of 0 is replaced by another mean pixel value.

It was quite natural to extend BTC to multi - spectrum images. Most color images are recorded in RGB space, which is perhaps the most well-known color space. As described previously, BTC divides the image to be coded into small blocks and code them one at a time. For single bitmap BTC of color image, a single binary bitmap the same size as the block is created and two colors are computed to approximate the pixels within the block. To create a binary bitmap in the RGB space, an Inter Band Average Image (IBAI) is first created and a single scalar value is found as the threshold value. The bitmap is then created by comparing the pixels in the IBAI with the threshold value.

\section{PROPOSED CBIR TECHNIQUES}

The paper proposes 'Mask Shape BTC' image retrieval techniques using four different gradient operators (Roberts, Sobel, Prewitt and Canny) using slope magnitude technique and BTC. The performance of proposed image retrieval methods is compared with 'Mask Shape' based CBIR techniques.

\subsection{Mask-Shape CBIR}

In 'Mask-Shape' CBIR the shape feature vector is formed by applying slope magnitude method on Gradient of image in vertical and horizontal directions. This feature vector is considered for finding the similarity of query image with database images. The problem of this technique is that all the images in the database must have same dimensions as that of query image. The selection of gradient operators like Robert, Sobel, Prewitt and canny results into four variations of 'Mask-Shape' based image retrieval.

\subsection{Mask-Shape-BTC CBIR}

The problem of having all the databse images with same size for image retrieval can be resolved using proposed 'Mask Shape BTC' based CBIR methods. Here firstly, the shape features of the image are extracted by applying slope magnitude method on gradients of the image in vertical and horizontal directions and then the BTC is applied on obtained 'Mask Shape' images to have a shape feature vector with constant size irrespective of size of the image considered. Even in 'Mask shape BTC' based image retrieval four variations are considered using different gradient operators.

\section{IMPLEMENTATION}

The discussed image retrieval methods are implemented using MATLAB 7.0 on Intel Core 2 Duo processor T8100 $(2.1 \mathrm{GHz})$ with 2 GB of RAM. To check the performance of proposed technique a database of 1000 variable sized images spread across 11 categories has been used.

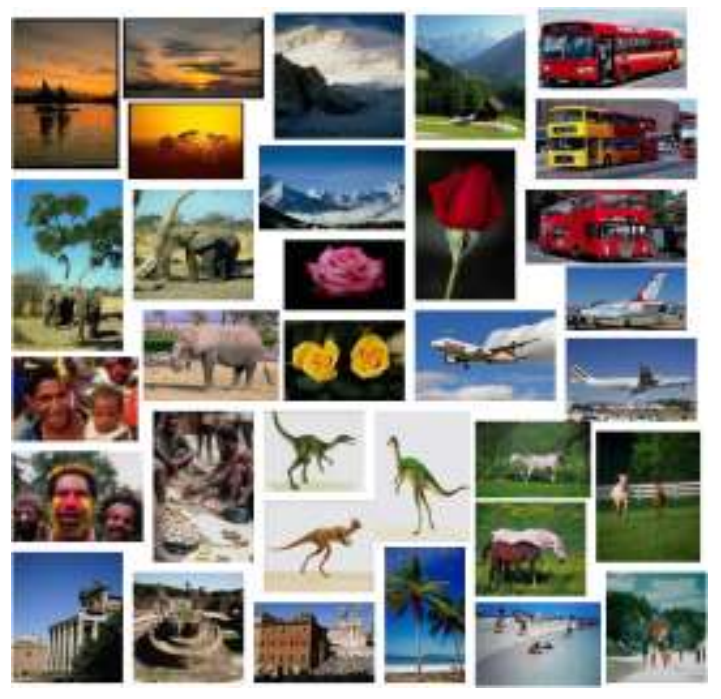

Figure 6: Sample Images from Generic Image Database [Image database contains total 1000 images with 11 categories]

Figure 6 shows sample image of generic image database. In all 55 queries (five from each category) are fired on the image database. The similarity between feature vectors is found using mean square error (MSE). The MSE between two feature vectors Vpi and Vqi can be computed as shown in equation 8 .

$$
M S E=\sum_{i=1}^{n}(V p i-V q i)^{2}
$$

To assess the retrieval effectiveness, we have used the precision and recall as statistical comparison parameters for our proposed technique of CBIR. The standard definitions of these two measures are given by following equations.

$$
\begin{gathered}
\text { Precision }=\frac{\text { Number of relevant images retrieved }}{\text { Total number of images retrieved }} \\
\text { Recall }=\frac{\text { Number of relevant images retrieved }}{\text { Total number of relevant images in database }}
\end{gathered}
$$




\section{RESULTS AND DISCUSSIONS}

For every discussed image retrieval technique, the values of average precision and average recall of all 55 queries are plotted against number of retrieved result images. Higher precision and recall values signifies better performance. The precision and recall curves for Mask-Shape CBIR techniques are shown in figure 7. Here Canny gradient operator based Mask-Shape image retrieval outperforms the other discussed techniques. Prewitt gives second best performance followed by Robert and Sobel. Figure 8 shows the performance comparison of Mask-Shape-BTC CBIR techniques based on discussed gradient operators. Here the performance comparison becomes difficult.

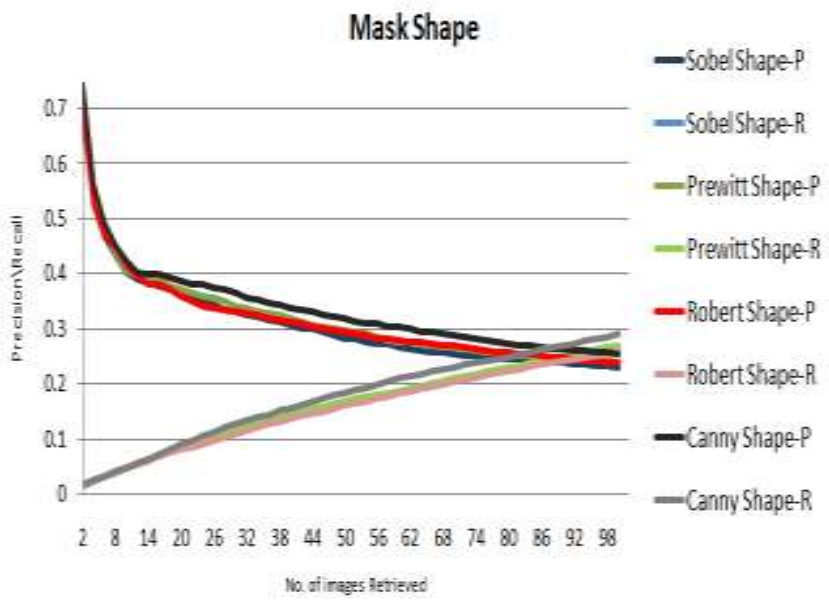

Figure 7: Precision and Recall for Mask-Shape CBIR techniques plotted against number of retrieved result images

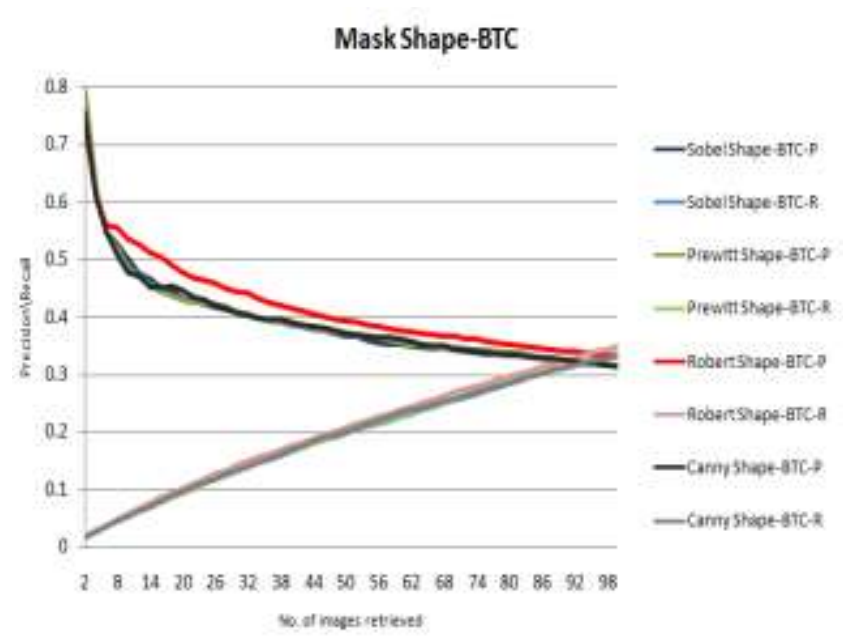

Figure 8: Precision and Recall for Mask-Shape-BTC CBIR techniques plotted against number of retrieved result images

The crossover point of precision and recall gives important performance measure for image retrieval techniques. Higher the crossover point is better will be the performance of CBIR method. Figure 9 gives the zoomed version of precision and recall curves for proposed CBIR techniques with the crossover point region enhanced. Here the Robert-Shape-BTC outperforms the other methods of image retrieval. All Shape-Mask-BTC based CBIR techniques gives better performance than Shape-Mask CBIR methods.

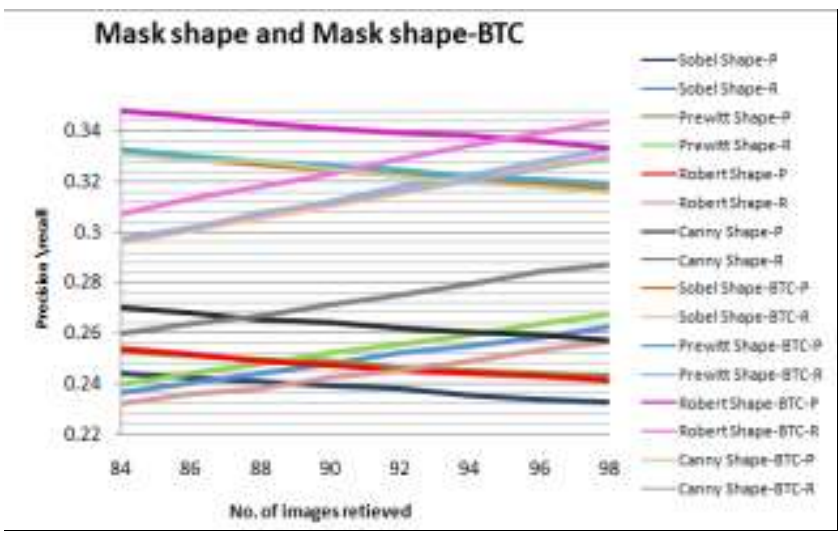

Figure 7: Crossover Points of Precision and Recall for discussed CBIR techniques

The values of crossover points of precision and recall for all proposed image retrieval methods are plotted in a bar chart as shown in figure 10. From this the conclusion can be confirmed that using BTC improves the performance of Mask-Shape image retrieval techniques. Among all Mask-Shape-BTC methods the Robert gradient operator performs the best followed by Prewitt.

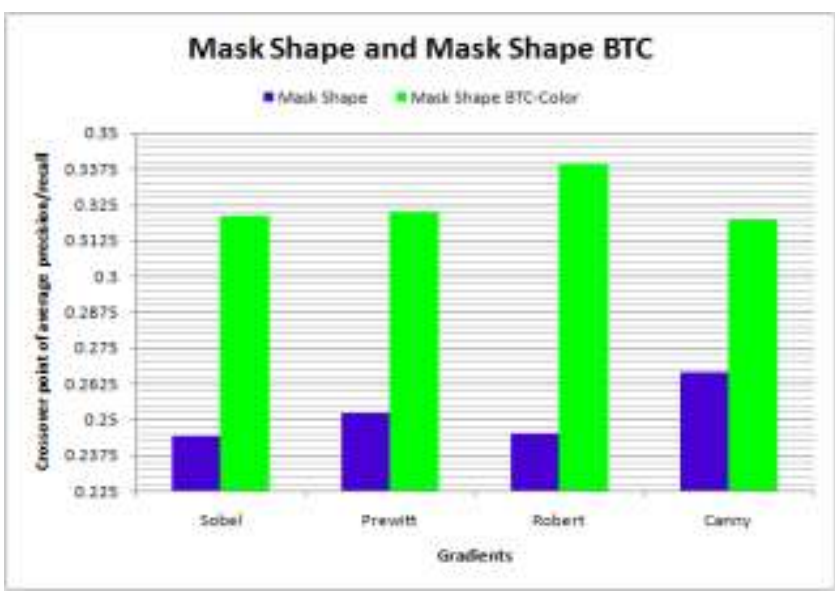

Figure 10: Performance comparison of discussed CBIR techniques

\section{CONCLUSION}

Image Retrieval is gaining momentum among researchers working in image processing and computer vision areas because of the wide number of applications. Image retrieval using shape features is the theme of work presented here. The shape features can be extracted using slope magnitude method applied on gradient of images taken in both horizontal and vertical directions. Using Roberts, Sobel, Prewitt and Canny gradient operators four variations in discussed Mask-Shape-CBIR methods can be obtained. The experimentation results show that the Canny gradient operator givers best performance in all Mask-ShapeCBIR techniques. The problem with these Mask-Shape-CBIR 
methods is the need of resizing the database images to match it with the size of query. This drawback is removed using proposed Mask-Shape-BTC-CBIR methods. In proposed image retrieval techniques the feature vectors are formed by applying the block truncation coding (BTC) on the shape image obtained using slope magnitude applied on gradient of the image in both horizontal and vertical direction. The higher precision and recall values obtained in experimentation prove that the proposed Mask-Shape-BTC image retrieval techniques outperform the conventional MaskShape based CBIR methods. The advantage of using BTC is that there would be no need of image resizing for feature vector extraction from image with improvement in performance of image retrieval results. In all the Robert-Mask-Shape-BTC gives the best performance.

\section{REFERENCES}

[1]. Dr.H.B.Kekre, Sudeep D. Thepade, "Boosting Block Truncation Coding using Kekre's LUV Color Space for Image Retrieval", WASET Int. Journal of Electrical, Computer and System Engineering (IJECSE), Volume 2, Number 3, pp. 172-180, Summer 2008. Available online at http://www.waset.org/ijecse/v2/v2-3-23.pdf

[2]. Dr.H.B.Kekre, Sudeep D. Thepade, "Image Retrieval using Augmented Block Truncation Coding Techniques", ACM International Conference on Advances in Computing, Communication and Control (ICAC3-2009), pp. 384-390, 23-24 Jan 2009, Fr. Conceicao Rodrigous College of Engg., Mumbai. Is uploaded on online ACM portal.

[3]. Dr.H.B.Kekre, Sudeep D. Thepade, "Rendering Futuristic Image Retrieval System", National Conference on Enhancements in Computer, Communication and Information Technology, EC2IT-2009, 20-21 Mar 2009, K.J.Somaiya College of Engg., Vidyavihar, Mumbai-77.

[4]. Dr.H.B.Kekre, Sudeep D. Thepade, "Improving 'Color to Gray and Back' using Kekre's LUV Color Space”, IEEE International Advanced Computing Conference 2009 (IACC'09), Thapar University, Patiala, INDIA, 6-7 March 2009. Is uploaded and available online at IEEE Xplore.

[5]. Dr.H.B.Kekre, Sudeep D. Thepade, "Color Traits Transfer to Grayscale Images", In Proc.of IEEE First International Conference on Emerging Trends in Engg. \& Technology, (ICETET-08), G.H.Raisoni COE, Nagpur, INDIA. Uploaded on online IEEE Xplore.

[6]. Image

Database

http://wang.ist.psu.edu/docs/related/Image.orig (Last referred on 23 Sept 2008)

[7]. Dr.H.B.Kekre, Sudeep D. Thepade, "Using YUV Color Space to Hoist the Performance of Block Truncation Coding for Image Retrieval", IEEE International Advanced Computing Conference 2009 (IACC'09), Thapar University, Patiala, INDIA, 6-7 March 2009.

[8]. Dr.H.B.Kekre, Sudeep D. Thepade, Archana Athawale, Anant Shah, Prathmesh Verlekar, Suraj Shirke,"Energy Compaction and Image Splitting for Image Retrieval using Kekre Transform over Row and Column Feature Vectors", International Journal of Computer Science and Network Security (IJCSNS), Volume:10, Number 1, January 2010, (ISSN: 1738-7906) Available at www.IJCSNS.org.

[9]. Dr.H.B.Kekre, Sudeep D. Thepade, "Image Retrieval using Color-Texture Features Extracted from Walshlet Pyramid",
ICGST International Journal on Graphics, Vision and Image Processing (GVIP), Volume 10, Issue I, Feb.2010, pp.9-18, www.icgst.com/gvip/Volume10/Issue1/P1150938876.html

[10]. Dr.H.B.Kekre, Sudeep D. Thepade, "Color Based Image Retrieval using Amendment Block Truncation Coding with YCbCr Color Space", International Journal on Imaging (IJI), Volume 2, Number A09, Autumn 2009, pp. 2-14. Available online at www.ceser.res.in/iji.html

[11]. Dr.H.B.Kekre, Tanuja Sarode, Sudeep D. Thepade, "ColorTexture Feature based Image Retrieval using DCT applied on Kekre's Median Codebook", International Journal on Imaging (IJI), Volume 2, Number A09, Autumn 2009,pp. 55-65. Available online at www.ceser.res.in/iji.html

[12]. Dr.H.B.Kekre, Sudeep D. Thepade, "Image Retrieval using Non-Involutional Orthogonal Kekre's Transform”, International Journal of Multidisciplinary Research and Advances in Engineering (IJMRAE), Ascent Publication House, 2009, Volume 1, No.I, pp 189-203, 2009. Abstract available online at www.ascent-journals.com

[13]. Dr.H.B.Kekre, Sudeep D. Thepade, "Improving the Performance of Image Retrieval using Partial Coefficients of Transformed Image", Int. Journal of Info. Retrieval, Serials Publications, Vol. 2, Issue 1, 2009, pp. 72-79

[14]. Dr.H.B.Kekre, Sudeep D. Thepade, Archana Athawale, Anant Shah, Prathmesh Verlekar, Suraj Shirke, "Performance Evaluation of Image Retrieval using Energy Compaction and Image Tiling over DCT Row Mean and DCT Column Mean", Springer-International Conference on Contours of Computing Technology (Thinkquest-2010), Babasaheb Gawde Institute of Tech., Mumbai, 13-14 March 2010, The paper will be uploaded on online Springerlink.

[15]. Dr.H.B.Kekre, Tanuja K. Sarode, Sudeep D. Thepade, Vaishali Suryavanshi,"Improved Texture Feature Based Image Retrieval using Kekre's Fast Codebook Generation Algorithm", Springer-International Conference on Contours of Computing Technology (Thinkquest-2010), Babasaheb Gawde Institute of Technology, Mumbai, 13-14 March 2010, The paper will be uploaded on online Springerlink.

[16]. Dr.H.B.Kekre, Sudeep D. Thepade, "Color Traits Transfer to Grayscale Images", IEEE Int. Conf. on Emerging Trends in Engg. and Technology, ICETET-2008, 16-18 July 2008.

[17]. Hirata K., Kato T. "Query by visual example - content based image retrieval", In Proc. of 3rd Int. Conf. on Extending Database Tech., EDBT'92, 1992, pp 56-71.

[18]. Sagarmay Deb, Yanchun Zhang, "An Overview of Content Based Image Retrieval Techniques," Technical Report, University of Southern Queensland.

[19]. Rafael C. Gonzalez, Richard E. Woods, "Digital Image Processing". Chapter 10, pg 599-607. Published by Pearson Education, Inc. 2005.

[20]. LI Xue-wei, ZHANG Xin-rong, "A Perceptual Color Edge Detection Algorithm". 2008 International Conference on Computer Science and Software Engineering.

[21]. William I. Grosky, "Image Retrieval - Existing Techniques, Content-Based (CBIR) Systems" Department of Computer and Information Science, University of Michigan-Dearborn, Dearborn, MI, USA, http://encyclopedia.jrank.org/articles/pages/6763/ImageRetrieval.html\#ixzz0130drFVs, (referred on 9 March 2010) 
[22]. Bill Green, "Canny Edge Detection Tutorial", 2002. http://www.pages.drexel.edu/ weg22/can_tut.html, referred on 9 March 2010

[23]. John Eakins, Margaret Graham, "Content Based Image Retrieval”, Chatpter 5.6, pg 36-40, University of Northrumbia at New Castle, October 1999

\section{Author Biographies}

Dr. H. B. Kekre has received B.E. (Hons.) in Telecomm. Engineering. from Jabalpur University in 1958, M.Tech (Industrial Electronics) from IIT Bombay in 1960, M.S.Engg. (Electrical Engg.) from University of Ottawa in 1965 and Ph.D. (System Identification) from IIT Bombay in $1970 \mathrm{He}$ has worked as Faculty of Electrical Engg. and then HOD Computer Science and Engg. at IIT Bombay. For 13 years he was working as a professor and head in the Department of Computer Engg. at Thadomal Shahani Engineering. College, Mumbai. Now he is Senior Professor at MPSTME, SVKM's NMIMS University. He has guided 17 Ph.Ds, more than 100 M.E./M.Tech and several B.E./B.Tech projects. His areas of interest are Digital Signal processing, Image Processing and Computer Networking. He has more than 320 papers in National / International Conferences and Journals to his credit. He was Senior Member of IEEE. Presently He is Fellow of IETE and Life Member of ISTE Recently ten students working under his guidance have received best paper awards and two have been conferred Ph.D. degree of SVKM's NMIMS University. Currently 10 research scholars are pursuing Ph.D. program under his guidance

Sudeep D. Thepade has Received B.E.(Computer) degree from North Maharashtra University with Distinction in 2003. M.E. in Computer Engineering from University of Mumbai in 2008 with Distinction, currently pursuing Ph.D. from SVKM's NMIMS, Mumbai. He has about than 07 years of experience in teaching and industry. He was Lecturer in Dept. of Information Technology at Thadomal Shahani Engineering College, Bandra(w), Mumbai for nearly 04 years. Currently working as Associate Professor in Computer Engineering at Mukesh Patel School of Technology Management and Engineering, SVKM's NMIMS University, Vile Parle(w), Mumbai, INDIA.
$\mathrm{He}$ is member of International Association of Engineers (IAENG) and International Association of Computer Science and Information Technology (IACSIT), Singapore. $\mathrm{He}$ is reviewer for many international journals and in the international advisory panel for many international conferences. His areas of interest are Image Processing and Computer Networks. $\mathrm{He}$ has about 75 papers in National/International Conferences/Journals to his credit with a Best Paper Award at Int. Conference SSPCCIN-2008, Second Best Paper Award at ThinkQuest-2009 National Level faculty paper presentation competition and Best Paper Award at Springer Int. Conf. ICCCT2010.

Shobhit Wadhwa is pursuing a B.Tech degree in Information Technology from MPSTME, SVKM's NMIMS University, Mumbai, India. His areas of interest lie in image processing and information systems development. He is also a member of the IEEE committee of his college.

Satyajit Singh is pursuing a B.Tech degree in Information Technology from MPSTME, SVKM's NMIMS University, Mumbai,India. His areas of interest lie in the fields of Image processing and Wireless technologies.

Priyadarshini Mukherjee is pursuing a B.Tech degree in Information Technology from MPSTME, SVKM's NMIMS University, Mumbai. Her interests lie in the fields of image processing and website development.

Miti Kakaiya is pursuing a B.Tech degree in Information Technology from MPSTME, SVKM's NMIMS University, Mumbai. Her interests lie in the field of Image processing and website designing. 